\title{
Perioperative, Single-Surgeon Experience in Cerebrovascular Bypass Procedures at Academic Medical Center and Community Hospital
}

\author{
Kathleen W. Nissman \\ Ali R. Zomorodi \\ Michael L. James 3,5 \\ ${ }^{1}$ American Anesthesiology of North Carolina, Raleigh, North \\ Carolina, United States \\ ${ }^{2}$ Department of Neurosurgery, Duke University, Durham, North \\ Carolina, United States \\ ${ }^{3}$ Department of Anesthesiology, Duke University, Durham, North \\ Carolina, United States \\ ${ }^{4}$ Department of Biostatistics, Duke University, Durham, North \\ Carolina, United States \\ ${ }^{5}$ Department of Neurology, Duke University, Durham, North \\ Carolina, United States
}

Dhanesh K. Gupta ${ }^{3}$

Ishwori Dhakal ${ }^{4} \quad \mathrm{Yi}-\mathrm{Ju} \mathrm{Li}^{4}$

J Neuroanaesthesiol Crit Care 2018;5:173-176

\begin{abstract}
Address for correspondence Michael L. James, MD, Department of Anesthesiology and Neurology, Duke University, DUMC 3094, Durham, NC 27710, United States (e-mail: michael.james@duke.edu).
\end{abstract}

\begin{abstract}
Keywords

- moyamoya

- extracranial intracranial bypass

- subspecialty care

- community hospital care

- neuroanesthesiology

- neurosurgery

Background Little evidence exists for superiority of neurosurgical outcomes from care subspecialization. Outcomes of a single neurosurgeon after complex vascular neurosurgery in an academic medical center were compared against those in a community hospital.

Methods In this retrospective analysis of extracranial-intracranial vascular bypass operations performed between July 1, 2013 and February 1, 2015, cases were identified by cross-referencing the electronic medical record with the surgeon's own records. Pre-, intra-, and postoperative variables were abstracted from cases performed at a tertiary center and a community hospital. Dichotomous postoperative data recorded included extubation in the operating room (OR), readmission, and survival to discharge, and length of stay was also analyzed. Due to small sample size and low readmission rate, Firth's penalized likelihood tests were incorporated in the logistic regression model for parameter estimation and testing.

Results A total of 28 hemispheres in 26 patients were included: 18 hemispheres in 16 patients at the tertiary center and 10 hemispheres in 9 patients at the community hospital. Differences were found in operative time (tertiary mean: $7.21+2.5$ hours, community mean: $5.19+0.9$ hours, $p=0.0074$ ) and readmission to the tertiary center $(p=0.078)$. However, significant difference was observed only for anesthetic type (more likely to include remifentanil and propofol at the tertiary center, $p=0.0104$ ). Conclusion Subspecialty care alone may be insufficient to enhance outcome after complex neurosurgical procedures.
\end{abstract}

\section{Introduction}

With respect to neurosurgical perioperative care, little evidence currently exists about the superiority of anesthetic subspecialization. While dedicated neurocritical care improves outcomes for acute intracranial hemorrhage and head trauma, evidence exists to the contrary for elective neurosurgical cases on patients with few comorbidities. ${ }^{1,2,3}$ received

February 21, 2018 accepted after revision June 7, 2018 published online July 3, 2018
DOI https://doi.org/

10.1055/s-0038-1666889. ISSN 2348-0548.
Copyright $\odot 2018$ Indian Society of Neuroanaesthesiology and Critical Care
License terms

(a) (1) $\ominus \circledast$ 
Moyamoya disease, or cerebral arterial steno-occlusive disease, is an occlusive cerebrovascular disease affecting the internal carotid artery. In patients receiving medical management of single hemispheric moyamoya disease diagnosed clinically, the 5-year risk of recurrent ipsilateral stroke is to be $65 \%{ }^{4}$ One treatment for cerebral arterial steno-occlusive disease is extracranial-intracranial (EC-IC) carotid artery bypass procedure. Direct EC-IC procedure, usually a superficial temporal artery to middle cerebral artery bypass, has been shown to have better outcomes when compared with an indirect bypass procedure (e.g., a superficial temporal artery to dural bypass). ${ }^{5}$ Surgical intervention by bypass procedure has been shown to reduce subsequent stroke and mortality risk to 17 to $22 \%$, whereas perioperative stroke risk is estimated at $13 \%$ per operated hemisphere. ${ }^{4,6}$

A unique situation exists in our health care system: a single vascular neurosurgeon practices at both an academic tertiary care hospital and an affiliated local community hospital. At the academic medical center, all perioperative care is subspecialized. Anesthesia is performed by subspecialty-trained neuroanesthesiologists, and postoperative care is performed by a dedicated neurocritical care service. At the community hospital, every anesthesia is performed by general anesthesiologists, and postoperative care is administered in a general medical or surgical intensive care unit. Thus, the outcomes of a single surgeon in a single-patient population after complex vascular neurosurgery in an academic medical center were compared against those in a regional community hospital.

\section{Materials and Methods}

All procedures were performed by single board-certified neurosurgeon specializing in neurovascular diseases. Anesthetics at the academic medical center were all performed by subspecialty-trained neuroanesthesiologists. All patients went directly to a neurosurgical intensive care unit (ICU) following the procedure. Anesthetics at the affiliated community hospital were performed by a private practice group; only one anesthesiologist in this group was a subspecialty-trained neuroanesthesiologist. All patients were admitted to a general medical or surgical ICU following the procedure at the affiliated community hospital.

Neurosurgical cases performed at the academic medical center are performed by eight neuroanesthesia subspecialty anesthesiologists. Patients are cared for in a 24-bed neurosurgical ICU staffed by a group of eight specialty-trained neurointensivists. The academic medical center itself is a tertiary and quaternary referral hospital with 938 beds. In contrast, the community hospital is staffed by a group of approximately 15 anesthesiologists with diverse subspecialty training, but all of whom practice as generalists. The hospital has 186 beds, and a single combined 15-bed medical or surgical ICU is staffed by a group of six intensivists with critical care certification through pulmonology or anesthesiology.

In this retrospective analysis of EC-IC bypass operations performed between July 1, 2013 and February 1, 2015, cases were identified by cross-referencing the electronic medical record with the surgeon's own records. All patients had a diagnosis of steno-occlusive carotid artery disease and were excluded if preoperative modified Rankin score was greater than 1 . The following preoperative variables were recorded: race, age, sex, and past medical historical data including those of transient ischemic attack, stroke, hypertension, diabetes, and tobacco abuse. Intraoperative data recorded for this analysis included inhalational agent, intravenous agent, antiseizure prophylaxis, mannitol dose, fluid, and urine output. Dichotomous postoperative data included extubation in the operating room (OR), readmission, and survival to discharge, and length of stay was also analyzed.

Descriptive statistics for each demographic and clinical variable were computed for each site where surgery was performed (academic medical center or community hospital). For categorical variables, we tested the frequency (percentage) differences between two clinical sites using chi-square or Fisher's exact tests. For continuous variables, we evaluated the normality of the data, first using Shapiro-Wilk test. Depending on the results of normality tests, two-sample $t$-tests were used to compare the mean differences of normally distributed continuous variables; otherwise, Wilcoxon Rank-Sum tests were used to compare the median differences between two clinical sites. To evaluate the factor associated with patient readmission, we conducted univariate logistic regression analysis for each covariate of interest. Due to the small sample size and the low readmission rate, Firth's penalized likelihood tests were incorporated in the logistic regression model for parameter estimation and testing. Variables meeting a nominal significance level $(p<0.05)$ were considered significant. All analyses were conducted using SAS 9.4 (Cary, North Carolina, United States).

This study was approved by the institutional review board and is Health Insurance Portability and Accountability Act compliant.

\section{Results}

A total of 28 hemispheres in 26 patients were included in this study: 18 hemispheres in 16 patients at the academic medical center and 10 hemispheres in 9 patients at the community medical center. The only two urgent, nonelective cases were performed at the academic medical center; these cases are excluded from analysis. The preoperative characteristics of these patients are noted in - Table $\mathbf{1}$. Notably, there were no statistically significant differences in baseline demographic data. The proportion of diabetics was greater at the academic site $(8 / 16)$ versus the community site $(1 / 10)$; this difference did not reach significance $(p=0.0873)$. There was no difference in the proportion of ASA (American Society of Anesthesiologists) classes 3 and 4 patients at each site.

Intra- and postoperative data are listed in - Table 2 . Significant differences were observed only for the intraoperative parameters OR time (academic mean: 7.21 hours, community mean: 5.19 hours, $p=0.0074$ ) and anesthetic use. Anesthetic use was more likely to include remifentanil and propofol at the academic medical center $(p=0.0104)$. Three of 16 cases used remifentanil and propofol alone, and 13 of 16 cases used a combination of a gas anesthetic plus remifentanil and 
Table 1 Demographic, perioperative, and outcome characteristics of extracranial-intracranial bypass procedures performed at an academic hospital and an affiliated community hospital

\begin{tabular}{|c|c|c|c|}
\hline & $\begin{array}{l}\text { Academic medical } \\
\text { center }(n=16)\end{array}$ & $\begin{array}{l}\text { Community hospital } \\
(n=10)\end{array}$ & $p$-Value \\
\hline Age (SD) & $48(13.1)$ & 43.5 (14.9) & 0.4263 \\
\hline Sex: Female & $13(81.25 \%)$ & $8(80 \%)$ & 1.0000 \\
\hline \multicolumn{4}{|l|}{ Race } \\
\hline Black & $9(56.25 \%)$ & $4(40 \%)$ & \multirow[t]{3}{*}{0.5044} \\
\hline Nonblack, non-Caucasian & $5(31.25 \%)$ & $3(30 \%)$ & \\
\hline Caucasian & $2(12.5 \%)$ & $3(30 \%)$ & \\
\hline Tobacco use & $4(25 \%)$ & $5(50 \%)$ & 0.2341 \\
\hline Hypertension & $12(75 \%)$ & $5(50 \%)$ & 0.2341 \\
\hline Diabetes & $8(50 \%)$ & $1(10 \%)$ & 0.0873 \\
\hline TIA & $10(62.5 \%)$ & $8(80 \%)$ & 0.4198 \\
\hline Stroke & $8(50 \%)$ & $8(80 \%)$ & 0.2177 \\
\hline Prior bypass & $3(18.75 \%)$ & $1(10 \%)$ & 1.0000 \\
\hline \multicolumn{4}{|l|}{ ASA class } \\
\hline 3 & $11(68.75 \%)$ & $6(60 \%)$ & \multirow[t]{2}{*}{0.6924} \\
\hline 4 & $5(31.25 \%)$ & $4(40 \%)$ & \\
\hline OR time, hours (SD) & $7.21(2.5)$ & $5.19(0.9)$ & 0.0074 \\
\hline Extubated at end of case & $12(75 \%)$ & $9(90 \%)$ & 0.4862 \\
\hline Readmission rate & $8(50 \%)$ & $1(10 \%)$ & 0.0873 \\
\hline Length of stay, days (SD) & $3.81(4.7)$ & $2.60(1.1)$ & 0.8223 \\
\hline Deaths & 1 & 0 & \\
\hline
\end{tabular}

Abbreviations: ASA, American Society of Anesthesiologists; OR, operating room; SD, standard deviation; TIA, transient ischemic attack.

Table 2 Perioperative characteristics of extracranial-intracranial bypass procedures performed at an academic hospital and an affiliated community hospital

\begin{tabular}{|c|c|c|c|}
\hline & $\begin{array}{l}\text { Academic medical } \\
\text { center }(n=16)\end{array}$ & $\begin{array}{l}\text { Community hospital } \\
(n=10)\end{array}$ & $p$-Value \\
\hline \multicolumn{4}{|l|}{ Anesthetic regimen } \\
\hline Volatile & 0 & $4(40 \%)$ & \multirow[t]{3}{*}{0.0104} \\
\hline Volatile + intravenous & $13(81.25 \%)$ & $6(60 \%)$ & \\
\hline Intravenous & $3(18.75 \%)$ & 0 & \\
\hline Crystalloid & $3,740.63(1,907.6)$ & $3,735.0(1693.5)$ & 0.9790 \\
\hline Urine output (SD) & $2,507.5(2166)$ & $2,081.0(1661.6)$ & 0.6924 \\
\hline \multicolumn{4}{|c|}{ Intraoperative medication use } \\
\hline Remifentanil & $15(93.75 \%)$ & $6(60 \%)$ & 0.0549 \\
\hline Propofol & $6(37.5 \%)$ & $2(20 \%)$ & 0.4198 \\
\hline Desflurane & $0(0 \%)$ & $2(20 \%)$ & 0.1385 \\
\hline Isoflurane & 9 (56.25\%) & $5(50 \%)$ & 0.7558 \\
\hline Sevoflurane & $4(25 \%)$ & $3(30 \%)$ & 1.0000 \\
\hline \multicolumn{4}{|l|}{ Antiepileptic use } \\
\hline Levetiracetam & $7(43.75 \%)$ & $6(60 \%)$ & 0.6882 \\
\hline Phosphenytoin & $2(12.5 \%)$ & $0(0 \%)$ & 0.5077 \\
\hline
\end{tabular}

Abbreviation: SD, standard deviation. 
propofol at the academic medical center. At the community hospital, 4 of 10 patients received gas-only anesthesia and 6 of 10 patients received gas plus remifentanil and propofol anesthesia. While not quite reaching statistical significance ( $p=0.0873$ ), readmission occurred for 8 of 16 patients at the academic medical center and for only 1 of 10 patients at the community hospital. In univariate analysis, readmission rates by hospital type nearly reached statistical significance $(p=$ 0.078). No difference was observed for length of stay or postoperative extubation status. The only death was recorded at the academic site.

When the two urgent cases were included in the analysis, the statistical outcomes were unchanged even though both of these patients were ASA class 4.

\section{Discussion}

This is the first study that examines the effect of the perioperative environment on the outcomes of a complex neurosurgical procedure in two settings: an academic medical center with subspecialized perioperative care and a community medical center without subspecialized perioperative care. No significant differences in outcome were observed.

Neurosurgical critical care subspecialization seems to be most valuable for patients with conditions having a high associated in-hospital mortality rate. For example, Mirski et $\mathrm{al}^{1}$ found that subspecialized neurocritical care reduced in-hospital mortality in intracranial hemorrhage patients from 36 to $19 \%$. When the expected mortality rate following elective surgeries is low, the value of subspecialized neurointensive care is less clear. ${ }^{2}$ In our series, there was only one (3.6\%) death.

Our study is limited in that death and readmission are our only endpoints. In fact, readmission rates may not be considered true predictors of successful operation in these complicated patients. While these patients suffered no difference in these infrequent outcomes, subtle neurocognitive changes may be occurring, which would require larger prospective study to detect. Of note, neurocognitive decline following successful EC-IC bypass may be small when measured by formal neurocognitive testing pre- and 6 months post-operation. ${ }^{7}$ Notably, quality-of-life data are also absent for our analyses and may supersede other more traditional outcome metrics from a patient or family perspective. Despite these limitations, these preliminary data raise interesting questions about performing complex neurosurgery. In the hands of a skilled surgeon, complex elective neurosurgical cases, in particular EC-IC bypass, may be safely performed at community hospitals. However, several confounders or limitations exist that may make this conclusion an overstatement: unconscious selection bias, close proximity of the tertiary care or academic hospital, and small sample size. Because our patient population experienced relatively good outcomes, confirmation bias may have influenced the selection of moyamoya patients. Although our neurosurgeon does not specifically assign patients to the academic medical center or the regional hospital based on the relative patient's morbidities, an unconscious selection bias may be present. The community hospital is part of a network that includes a tertiary medical center located 45 minutes away, which may function as a "safety net" for case selection. Postoperative transfers to the tertiary care hospital for subspecialized care did not occur in this specific group of patients, but they have occurred for this surgeon's patients at other times. The study is further limited by small sample size.

This work raises interesting clinical questions for groups wishing to replicate the single surgeon at dual-site model; however, any arrangement should be carefully considered. Evaluation of cost benefits of this arrangement may help maximize health system efficiency, including scheduling of cases. Further study into the economic system wide benefits may make this practice more appealing for implementation.

\section{Funding}

This study was funded by the Department of Anesthesiology, Duke University, Durham, North Carolina, United States.

\section{Conflict of Interest}

None. The manuscript has been read and approved by all authors.

\section{Acknowledgments}

The authors would like to acknowledge Kathy Gage for her assistance with medical editing. This work was supported by the Department of Anesthesiology at Duke University.

\section{References}

1 Mirski MA, Chang CW, Cowan R. Impact of a neuroscience intensive care unit on neurosurgical patient outcomes and cost of care: evidence-based support for an intensivistdirected specialty ICU model of care. J Neurosurg Anesthesiol 2001;13(2):83-92

2 Terada Y, Inoue S, Tanaka Y, Kawaguchi M, Hirai K, Furuya H. The impact of postoperative intensive care on outcomes in elective neurosurgical patients in good physical condition: a single centre propensity case-matched study. Can J Anaesth 2010;57(12):1089-1094

3 Varelas PN, Eastwood D, Yun HJ, et al. Impact of a neurointensivist on outcomes in patients with head trauma treated in a neurosciences intensive care unit. J Neurosurg 2006;104(5):713-719

4 Hallemeier CL, Rich KM, Grubb RL Jr, et al. Clinical features and outcome in North American adults with moyamoya phenomenon. Stroke 2006;37(6):1490-1496

5 Houkin K, Kamiyama H, Abe H, Takahashi A, Kuroda S. Surgical therapy for adult moyamoya disease. Stroke 1996;27(8):1342-1346

6 Chiu D, Shedden P, Bratina P, Grotta JC. Clinical features of moyamoya disease in the United States. Stroke 1998;29(7):1347-1351

7 Inoue T, Ohwaki K, Tamura A, Tsutsumi K, Saito I, Saito N. Postoperative transient neurological symptoms and chronic subdural hematoma after extracranial-intracranial bypass for internal carotid/middle cerebral atherosclerotic steno-occlusive diseases: negative effect on cognitive performance. Acta Neurochir (Wien) 2016;158(1):207-216 